\title{
Combi-lipase for heterogeneous substrates: a new approach for hydrolysis of soybean oil using mixtures of biocatalysts
}

\author{
Joana S. Alves, ${ }^{a}$ Nathália S. Vieira, ${ }^{a}$ Alisson S. Cunha, ${ }^{a}$ Alexandre M. Silva, ${ }^{a}$ \\ Marco A. Záchia Ayub, ${ }^{a}$ Roberto Fernandez-Lafuente ${ }^{b}$ and Rafael C. Rodrigues ${ }^{\star a}$
}

\begin{abstract}
The concept of the combi-lipase biocatalyst has been proposed. It is based on the combination of different lipases as biocatalysts in reactions using heterogeneous substrates. The hydrolysis of soybean oil was evaluated as a model substrate, and Novozym 435 (CALB), Lipozyme TL-IM (TLL), and Lipozyme RM-IM (RML) were used as biocatalysts. Results showed that, although individually TLL was the most active enzyme, whereas CALB was the less active one, the combination of $80 \%$ of RML and $20 \%$ of CALB was the best biocatalyst. Reaction parameters were optimized, allowing to obtain more than $80 \%$ hydrolysis in $24 \mathrm{~h}$ using the combi-lipase, up from less than $50 \%$ with any individual lipase. Reusability of the combi-lipase showed that it could be used for at least 15 cycles without any significant decrease. The concept of the combibiocatalyst might be a useful technology for reactions including full modification of heterogeneous substrates.
\end{abstract}

Received 21st October 2013 Accepted 2nd January 2014

DOI: 10.1039/c3ra45969a

www.rsc.org/advances the main fatty acid accounts for no more than 70 or $80 \%$ of the oil nature, usually much less, meaning that there is an heterogeneous mixture of triglycerides. Other problems that will slow down hydrolyses are the production of diglycerides that may be not easily recognized by the used lipase and fatty acids inhibition. Finally, during oil hydrolysis the reaction $\mathrm{pH}$ is generally kept uncontrolled to prevent saponification and to avoid problems during purifications steps, thus, in conclusion, reaction conditions will be heterogeneous and will be changing along the reaction course. Therefore, it could be hypothesized that the full hydrolysis of complex substrates such as vegetable oils, could be better performed using a mixture of biocatalysts made up of different enzymes, with different specificities and activities. It was shown, for instance, that the combined use of 2 different 1,3-specific lipases from Rhizomucor miehei (RML) and Thermomyces laguginosus (TLL), improved the reaction rate and the yield of the synthesis of biodiesel using soybean oil as substrate. ${ }^{15}$

Some interesting lipases are commercially available. The probably most used biocatalyst by industry is Novozym 435, an immobilized preparation of the lipase B from Candida antarctica (CALB) on the hydrophobic resin Lewatit VP OC $1600 .^{\mathbf{1 6}}$ Lipozyme TL-IM is another widely used lipase, originally produced by Thermomyces lanuginosus (TLL), but industrially obtained from a genetically modified strain of Aspergillus ory$z a e^{17,18}$ TLL was immobilized on a cationic silicate via anion exchange $^{\mathbf{1 9 , 2 0}}$ and it has been used in multiple reactions. ${ }^{21}$ Finally, Lipozyme RM-IM is prepared by the immobilization of the lipase from Rhizomucor miehei (RML) on Duolite ES 562, which is a weak anion-exchange resin based on phenol-formaldehyde copolymers. ${ }^{22-24}$ RML has been reviewed for its uses, from chemical processes ${ }^{25}$ to oils modification. ${ }^{26}$

\footnotetext{
aiotechnolgy, Bioprocess and Biocatalysis Group, Food Science and Technology Institute, Federal University of Rio Grande do Sul, Av. Bento Gonçalves, 9500, P.O. Box 15090, ZC 91501-970, Porto Alegre, RS, Brazil. E-mail: rafaelcrodrigues@ufrgs.br; Web: www.ufrgs.br/bbb; Fax: +55 513308 7048; Tel: +55 5133087793

${ }^{b}$ Department of Biocatalysis, ICP - CSIC. Campus UAM-CSIC. Cantoblanco, ZC 28049, Madrid, Spain
} 
In this context, the aim of this research was to test the enzymatic hydrolysis of oils based on the design of a "combi-lipase biocatalyst" formed by the mixture of the three most commonly used immobilized lipases Novozym 435, Lipozyme RM-IM, and Lipozyme TL-IM. As model substrate, it was chosen soybean oil, the most abundant and one of the cheapest vegetable oils, which has a heterogeneous composition of fatty acids. Central composite design and response surface methodology ${ }^{27}$ were used in order to optimize reaction parameters, whereas reusability of the biocatalyst was tested in several batch reactions.

\section{Material and methods}

\subsection{Enzymes and other materials}

Lipases from T. lanuginosus (TLL, Lipozyme TL-IM), R. miehei (RML, Lipozyme RM-IM) and C. antarctica (CALB, Novozym 435) were kindly donated by Novozymes (Novozymes, Spain). The enzymes were in their immobilized form; TLL was immobilized on a silicate support, RML on an anion-exchange resin, and CALB on a macroporous resin. Refined soybean oil was purchased at a local market, with a reported composition of (as mass fraction): palmitic acid $(11.9 \%)$, palmitoleic acid (0.3\%), stearic acid (4.1\%), oleic acid (23.2\%), linoleic acid (54.2\%), and linolenic acid $(6.3 \%)$. All other chemicals were of analytical or HPLC grade.

\subsection{Methods}

Except for the experimental design, all the experiments in this research were carried out as triplicates and the calculated standard error was always under 5\%.

2.2.1. Hydrolysis of oil. Different molar ratios of water were added to $5 \mathrm{mmol}$ of soybean oil into $50 \mathrm{~mL}$ Erlenmeyer flasks, added of varying concentrations of biocatalysts (TLL, RML, and CALB), according to the experimental design. The mixtures of soybean oil, water, and lipases were stirred in an orbital shaker (200 rpm) for the specific time and temperature. For each point of the experimental design or time course reactions, samples were collected at the desired times to measure the hydrolysis degree. The progress of hydrolysis was monitored by determination of the free fatty acid released by titration of $0.3 \mathrm{~g}$ samples using $0.01 \mathrm{M} \mathrm{NaOH}$ using phenolphthalein as $\mathrm{pH}$ indicator and $5 \mathrm{~mL}$ of ethanol as quenching agent.

2.2.2. Reactions using the combination of different lipases. In order to determine the optimal combination of lipases for the hydrolysis reaction, a 3-factor mixture design and triangular surface analysis was performed. The simplex-centroid design with interior points composed of 10 experiments is shown in Table 1 . The reaction conditions were: substrate molar ratio, $3: 1$ (water : oil); temperature, $40{ }^{\circ} \mathrm{C}$; biocatalyst content $10 \%$ (as the oil mass); and the reaction time was of $4 \mathrm{~h}$. The biocatalyst content corresponds to individual or mixtures of lipases according to Table 1.

2.2.3. Central composite design. After selecting the best lipase mixture, a central composite design of 3 variables was carried out in order to obtain the optimal conditions for the hydrolysis reaction. The variables and their coded and uncoded values are presented in Table 2. Table 3 shows 18 treatments of
Table 1 Experiments performed in the mixture design

\begin{tabular}{lllll}
\hline Experiment & TLL & RML & CALB & Conversion (\%) \\
\hline 1 & 1.000 & 0.000 & 0.000 & 21.40 \\
2 & 0.000 & 1.000 & 0.000 & 30.30 \\
3 & 0.000 & 0.000 & 1.000 & 4.61 \\
4 & 0.500 & 0.500 & 0.000 & 21.79 \\
5 & 0.500 & 0.000 & 0.500 & 13.45 \\
6 & 0.000 & 0.500 & 0.500 & 23.87 \\
7 & 0.333 & 0.333 & 0.333 & 22.44 \\
8 & 0.667 & 0.167 & 0.167 & 24.42 \\
9 & 0.167 & 0.667 & 0.167 & 25.04 \\
10 & 0.167 & 0.167 & 0.667 & 24.68
\end{tabular}

Table 2 Process variables and their levels used in the CCD

\begin{tabular}{|c|c|c|c|c|c|c|}
\hline \multirow[b]{2}{*}{ Variables } & \multirow[b]{2}{*}{ Name } & \multicolumn{5}{|c|}{ Coded levels } \\
\hline & & -1.68 & -1 & 0 & 1 & 1.68 \\
\hline$X_{1}$ & Temperature $\left({ }^{\circ} \mathrm{C}\right)$ & 30 & 36 & 45 & 54 & 60 \\
\hline$X_{2}$ & $\begin{array}{l}\text { Biocatalyst content } \\
\text { (\% relative to the } \\
\text { oil mass) }\end{array}$ & 5 & 9 & 15 & 21 & 25 \\
\hline$X_{3}$ & $\begin{array}{l}\text { Substrate molar } \\
\text { ratio (water : } \\
\text { soybean oil) }\end{array}$ & $3: 1$ & $4.8: 1$ & $7.5: 1$ & $10.2: 1$ & $12: 1$ \\
\hline
\end{tabular}

the 3 variables, each at 5 levels. The design was constructed of 8 factorial points, 6 axial points ( 2 axial points on the axis of design variable), and 4 replications at the central point. In each case, the percentage of conversion for hydrolysis was determined after $4 \mathrm{~h}$. The second-order polynomial equation for the variables is as follows:

$$
Y=\beta_{0}+\sum \beta_{\mathrm{i}} X_{\mathrm{i}}+\sum \beta_{\mathrm{ij}} X_{\mathrm{i}} X_{\mathrm{j}}+\sum \beta_{\mathrm{ii}} X_{\mathrm{i}}
$$

Table 3 Experimental design and results of the CCD

\begin{tabular}{lrrrl}
\hline & & & & $\begin{array}{l}\text { Hydrolysis conversion } \\
\text { Treatment }\end{array}$ \\
\hline 1 & $X_{1}$ & $X_{2}$ & $X_{3}$ & \\
\hline & -1 & -1 & -1 & 33.57 \\
3 & -1 & -1 & 1 & 40.03 \\
4 & -1 & 1 & -1 & 41.96 \\
5 & -1 & 1 & 1 & 35.23 \\
6 & 1 & -1 & -1 & 55.01 \\
7 & 1 & -1 & 1 & 51.88 \\
8 & 1 & 1 & -1 & 49.70 \\
9 & 1 & 1 & 1 & 57.95 \\
10 & -1.68 & 0 & 0 & 30.35 \\
11 & 1.68 & 0 & 0 & 42.92 \\
12 & 0 & -1.68 & 0 & 36.36 \\
13 & 0 & 1.68 & 0 & 41.41 \\
14 & 0 & 0 & -1.68 & 42.31 \\
15 (C) & 0 & 0 & 1.68 & 53.96 \\
16 (C) & 0 & 0 & 0 & 49.40 \\
17 (C) & 0 & 0 & 0 & 48.94 \\
18 (C) & 0 & 0 & 0 & 49.49 \\
& 0 & 0 & 0 & 51.14 \\
\hline & & & &
\end{tabular}


where $Y$ is the response variable, $\beta_{0}$ the constant, $\beta_{\mathrm{i}}, \beta_{\mathrm{ii}}, \beta_{\mathrm{ij}}$ are the coefficients for the linear, quadratic, and for the interaction effects, respectively, and $X_{\mathrm{i}}$ and $X_{\mathrm{j}}$ the coded levels of variables $x_{\mathrm{i}}$ and $x_{\mathrm{j}}$. The above quadratic equation was used to plot surfaces for all variables.

2.2.4. Statistical analysis. The experimental design and analysis of results were carried out using Statistica 7.0 (Statsoft, USA). The statistical analysis of the model was performed as analysis of variance (ANOVA). The significance of the regression coefficients and the associated probabilities, $p(t)$, were determined using the Student's $t$-test; the second order model equation significance was determined using the Fisher's $F$-test. The variance explained by model was given by the multiple determination coefficients, $R^{2}$. For each variable, the quadratic models were represented as contour plots (2D).

2.2.5. Enzyme reuse. After the hydrolysis reaction, the immobilized enzymes were separated from the reaction medium by vacuum filtration using a sintered glass funnel. The biocatalyst was washed 3 times with 5 volumes of n-hexane and the solvent was eliminated by incubation for $24 \mathrm{~h}$ at $25^{\circ} \mathrm{C}$.

\section{Results and discussion}

\subsection{Selection of the best combi-lipase biocatalyst for soybean oil hydrolysis}

Combination of different enzymes is mainly used for cascade or sequential reactions, however, in the present work, is being proposed the design of a combi-lipase biocatalyst strategy for the simultaneous hydrolysis of a mixture of different substrates. In Fig. 1 is shown the independent hydrolytic activities of the 3 selected immobilized lipases. TLL and RML, both 1,3-regio specific lipases, ${ }^{21,26}$ were more efficient, showing similar activities, whereas CALB, a non-specific lipase,$^{28}$ presented a slightly lower activity.

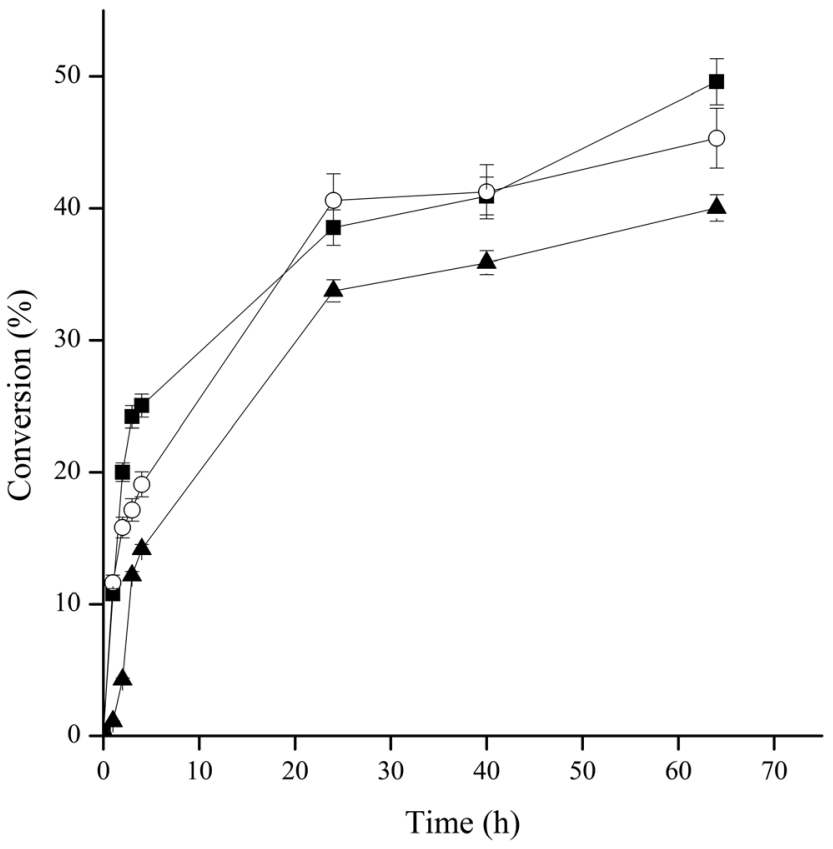

Fig. 1 Time course of hydrolysis of soybean oil catalyzed by ( $\mathbf{\square})$ TLL, (O) RML, and ( $\mathbf{\Delta}$ ) CALB. Reaction conditions: substrate molar ratio, 3: 1 water : soybean oil; biocatalyst content, $10 \%$ by oil mass; $40{ }^{\circ} \mathrm{C}$.

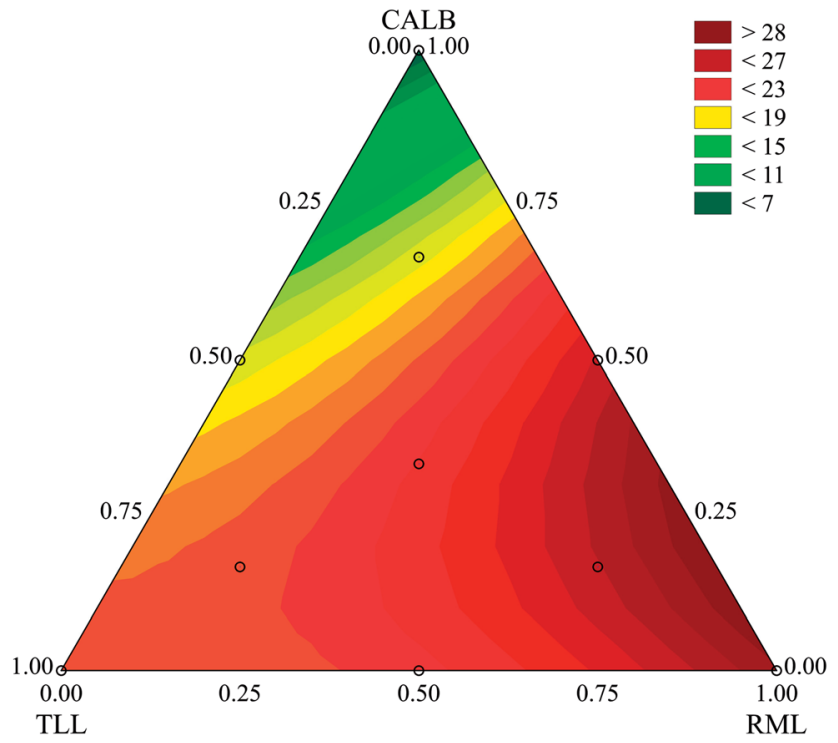

Fig. 2 Triangular surface for the mixture design. Reaction conditions: substrate molar ratio, 3:1 water: soybean oil; biocatalyst content, $10 \%$ by oil mass; $40{ }^{\circ} \mathrm{C} ; 4 \mathrm{~h}$.

Thus, in order to find the best combination of these enzymes, it was performed a 3-factor simplex-centroid design to found the ideal combi-lipase biocatalyst for the hydrolysis of soybean oil. The results obtained for the mixtures design are shown in Table 1, and graphically represented in Fig. 2. The lowest conversions were obtained using CALB alone, whereas the highest conversions were observed when higher amounts of RML were used. Mixtures of RML and CALB improved the activity, but this behavior was not observed for mixtures of TLL and CALB, or for TLL and RML. Thus, it is possible to propose that the best combilipase biocatalyst (among that studied) for hydrolysis of soybean oil is the combination of $80 \%$ of RML and $20 \%$ of CALB.

When used as a single enzyme, both TLL and RML produced the highest activities, their combination, however, did not improve the hydrolysis rate, probably because their similar substrate specificities. However, when CALB, the enzyme showing the lowest activity when used alone, combined in a mixture with RML, the resulting combi-biocatalyst improved the conversion rate by $50 \%$, when compared to the use of RML alone. In a previous report, ${ }^{15}$ the mixture of $65 \%$ of TLL and $35 \%$ of RML was found to be the more effective biocatalysts for soybean oil hydrolysis. Under the optimal reaction conditions for the mixture, it was obtained around $70 \%$ of hydrolysis in $4 \mathrm{~h}$ in that work. The differences might be explained by the diverse TLL preparations, with TLL covalently immobilized on Lewatit support activated with aldehyde groups, ${ }^{15}$ whereas in the present study it was used the commercial TLL form (Lipozyme TL-IM), which is immobilized by adsorption in an anion exchange matrix. ${ }^{19,20}$ These differences regarding nature of support and immobilization protocols are known to greatly affect the enzymes activities. ${ }^{\mathbf{2 9 - 3 2}}$

In the next experiments, the hydrolysis of soybean oil was optimized using the combi-lipase biocatalyst composed of $80 \%$ of RML and $20 \%$ of CALB. 


\subsection{Hydrolysis optimization}

3.2.1. Model fitting and ANOVA. A CCD was carried out to evaluate the reaction temperature, combi-lipase biocatalyst content, and substrate molar ratio (water : soybean oil), and the results are presented in Table 3 . The highest hydrolysis conversion was $57.95 \%$ obtained for treatment $8\left(54{ }^{\circ} \mathrm{C} ; 21 \%\right.$ of enzyme relative to oil mass; 10.2 : 1 water : soybean oil molar ratio). The experimental data have been adjusted to the proposed model in eqn (1) and the second-order polynomial model to hydrolysis reaction is presented in eqn (2).

$$
\begin{aligned}
Y= & 49.49+6.21 X_{1}-3.55 X_{1}{ }^{2}+0.94 X_{2}-2.76 X_{2}^{2} \\
& +1.79 X_{3}+0.51 X_{3}^{2}
\end{aligned}
$$

where $Y$ is the percentage of conversion for hydrolysis reaction, and $X_{1}, X_{2}$, and $X_{3}$, are the coded values of temperature, combilipase biocatalyst content, and substrate molar ratio, respectively.

The computed F-value (3.01) was statistically significant ( $p=$ $0.017)$. The goodness of the model was checked by the determination coefficient $\left(R^{2}=0.77\right)$ and correlation coefficient $(R=$ 0.88 ) showing a satisfactory representation of the process model and a good correlation between the experimental results and the theoretical values predicted by the model equation.

3.2.2. Effect of parameters on the hydrolysis rates. The linear effects of the variables on the hydrolysis rate were: temperature, 12.43; combi-biocatalyst, 1.88; substrate molar ratio, 3.58. All 3 variables presented positive effects, meaning that changing the variable level from -1 to 1 the response was increased. Temperature was the variable showing the highest effect, while the amount of biocatalyst was the lowest. Comparing the experiments where the only change in reaction conditions was the reaction temperature from $\left(36\right.$ or $54{ }^{\circ} \mathrm{C} ; 1-5$, $2-6,3-7,4-8)$, it can be observed that the hydrolysis rate increased almost 1.5-fold along with the temperature. Increasing temperature improves the enzymatic activity because of higher solubility of oil and its mobility on the porous support. The interactions between variables and their effects on hydrolysis rate are presented in the series of contour plots depicted in Fig. 3 , which were generated from the predicted model. Fig. 3a clearly shows the positive effect of temperature, the optimal being around $54{ }^{\circ} \mathrm{C}$, whereas the best amount of combi-lipase biocatalyst was close to the central value. The latter was the variable presenting the lowest effect of all. The interactions between substrate molar ratio with the amount of biocatalyst (Fig. 3b), and with temperature (Fig. 3c) strongly suggest that increasing the water content positively affects the hydrolysis rate. Water, which is a substrate of this reaction, is an important factor to keep the enzyme activity and stability. Temperature and amount of biocatalyst showed a V-shaped behavior where at lower substrate molar ratio level, their effects were more pronounced, and at higher water levels the range of temperature and biocatalyst content to obtain the maximal hydrolysis was wider.

3.2.3. Optimal conditions for hydrolysis and model validation. The optimal conditions for the hydrolysis reaction catalyzed using the mixture of RML and CALB (80\% RML and $20 \%$ CALB) were found to be $53{ }^{\circ} \mathrm{C}, 16 \%$ of combi-lipase
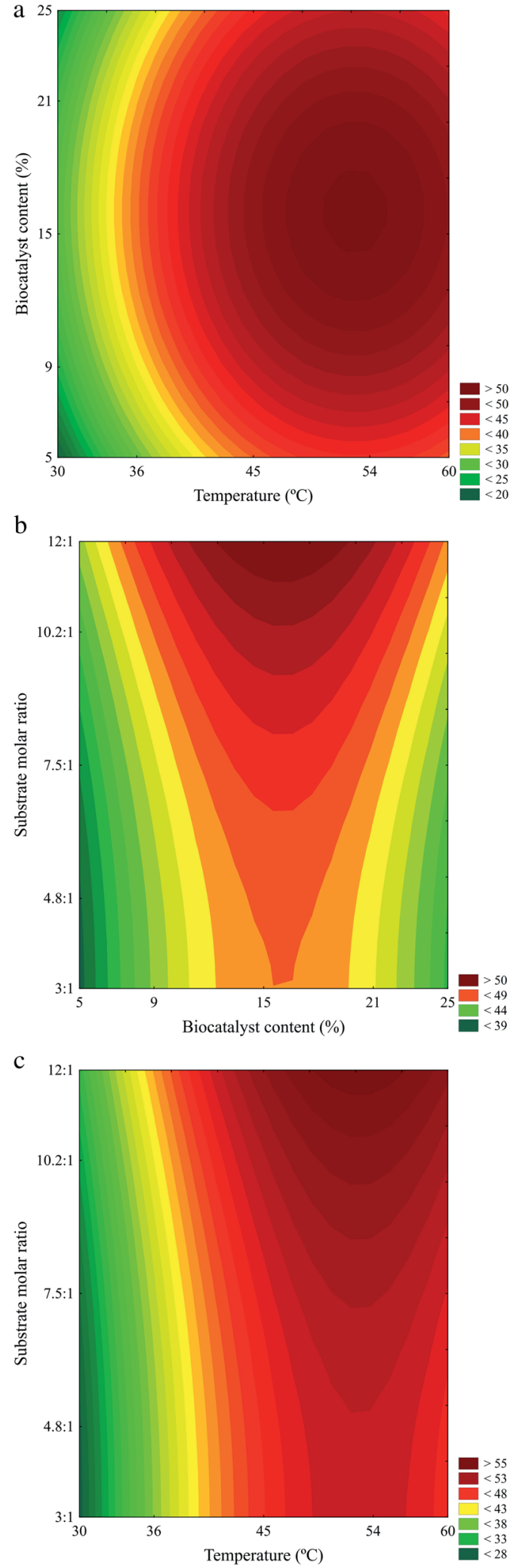

Fig. 3 Contour plots for conversion of hydrolysis of soybean oil. (a) Temperature versus biocatalyst content; (b) biocatalyst content versus substrate molar ratio; (c) temperature versus substrate molar ratio. In each figure, the missing variable was fixed at the central point. 
biocatalyst relative to oil mass, and a molar ratio of $12: 1$ water : soybean oil. Under these conditions the theoretical value for the hydrolysis rate of the reaction predicted by the model after $4 \mathrm{~h}$ is $57.9 \%$. Experimental validation of the proposed model was conducted under optimized conditions with four repetitions and the average hydrolysis rate obtained was $60.4 \pm$ $3.2 \%$, showing an excellent correlation between experimental results and the statistically predicted by the model.

\subsection{Time course of soybean oil hydrolysis}

The comparison of soybean oil hydrolysis carried out using combi-lipase ( $80 \%$ RML and $20 \%$ CALB) or the specific lipases used alone (TLL, RML and CALB), is presented in Fig. 4. In these experiments, the reactions were performed under the optimal conditions defined by the CCD, thus the performances of the individual lipases were slightly better than those represented in Fig. 1. The combi-lipase biocatalyst was significantly better than individual application of lipases, being 30\% higher than TLL, $35 \%$ higher than RML and 40\% higher than CALB, suggesting that RML and CALB have indeed different specificities regarding the fatty acids forming the glycerides. The results for the combilipase biocatalysts were also better than for other lipases. Sharma et $a .^{8}{ }^{8}$ reported the hydrolysis of cod liver oil by Candida cylindracea lipase. The authors obtained $26.9 \%$ of FFA yield in their most suitable conditions after $1 \mathrm{~h}$, while we reached to $35 \%$ in 1 h. Yigitoglu and Temoçin ${ }^{33}$ performed the hydrolysis of different vegetable oils catalyzed by lipase from Candida rugosa immobilized on glutaraldehyde-activated polyester fibers, obtaining as maximum less than $45 \mathrm{mg}$ of fatty acids after $5 \mathrm{~h}$, while in this work it was reached to around $3500 \mathrm{mg}$ in $5 \mathrm{~h}$.

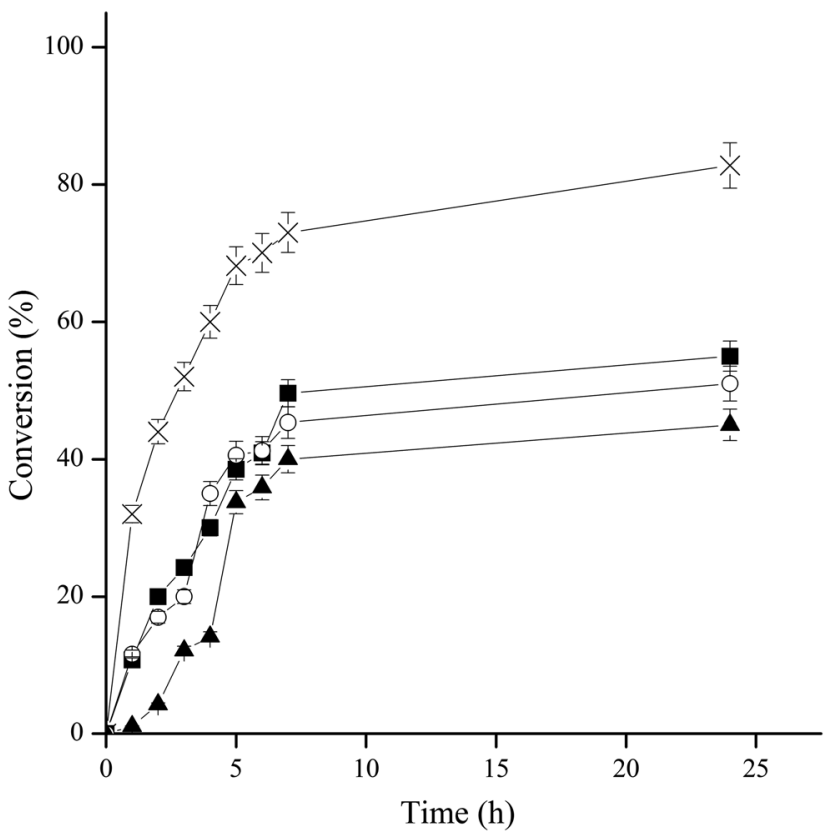

Fig. 4 Time course of hydrolysis of soybean oil catalyzed by ( $\mathbf{\square})$ TLL, (O) RML, ( $\mathbf{\Delta})$ CALB, and $(x)$ combi-lipase biocatalyst. Reaction conditions: substrate molar ratio, $12: 1$ water : soybean oil; enzyme content, $16 \%$ by oil mass; $53{ }^{\circ} \mathrm{C}$.
Moreover, these authors stated that the different degree of hydrolysis for each oil is due to impurities or the physical structure of the oil. Nevertheless, as discussed before, it is important to bear in mind that vegetable oils are a mixture of complex substrates formed by triglycerides, and as it was demonstrated, the difference in the hydrolysis degree may be mainly due to the specificity of each lipase to each fatty acid. Rathod and Pandit ${ }^{34}$ in the hydrolysis of different vegetable oils (castor, olive and coconut oils) catalyzed by lipolase, soluble preparation of T. lanuginosus, obtained as maximum yield less than $50 \%$ after $12 \mathrm{~h}$. Additionally, these authors concluded that as higher the unsaturation degree of the oil as higher the degree of hydrolysis, which reinforce our idea that the lipase specificity is the main point to be observed in hydrolysis reaction and that mixture of lipases as the combi-lipase biocatalysts will be better than individual lipases.

\subsection{Enzyme reuse}

The industrial applications of biocatalysts require enzymes stabilities in the reaction medium, allowing several batches reactions. Therefore, the combi-lipase biocatalyst was submitted to several hydrolyses batches under the optimal conditions in order to check the viability of a repeated process. In between each batch, it was performed a wash with $n$-hexane because it has been reported in other works ${ }^{35,36}$ that this solvent is very effective to remove any kind of substrate or product remaining after biocatalyst separation, consequently improving the biocatalyst reusability. The results for the repeated batches are presented in Fig. 5, showing that it was possible to use the combilipase biocatalyst for at least 15 batches keeping over $90 \%$ of its initial activity, suggesting that both enzymes retained their activities. It is important to remark that in this case both biocatalysts has to present operational stability. In other works, Lee

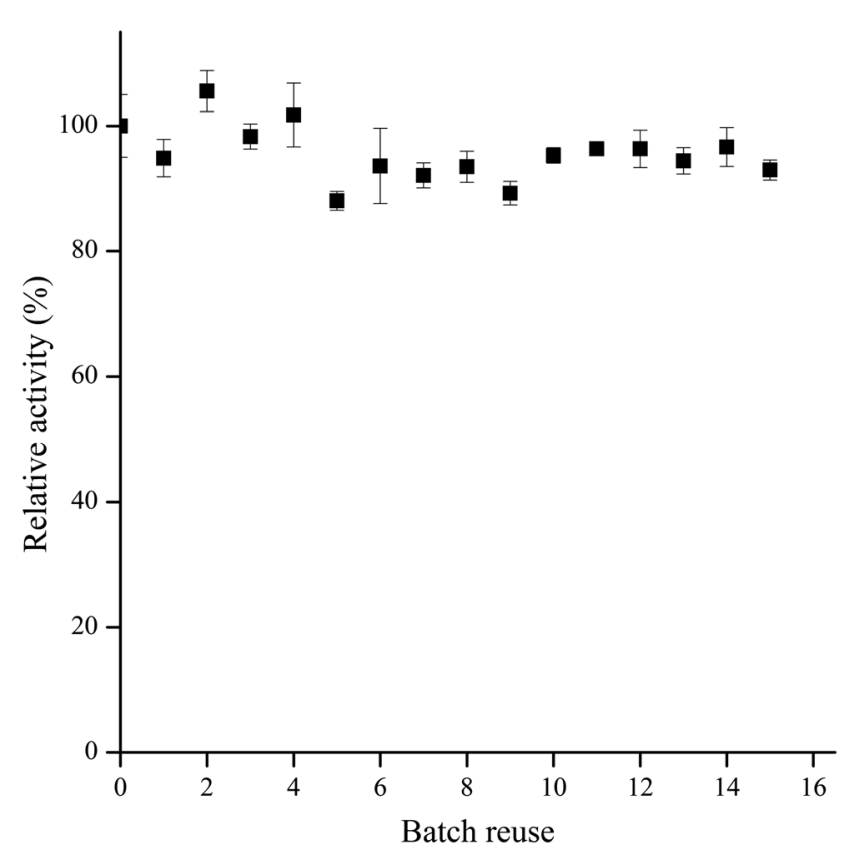

Fig. 5 Enzyme stability over repeated batches of hydrolysis of soybean oil catalyzed by the combi-lipase biocatalyst. 
et $a .^{14}$ reported a decrease of $20 \%$ of the initial activity of the mixture of $R$. oryzae and $C$. rugosa lipases in biodiesel synthesis after 5 uses. For individual enzymes, lipase from C. rugosa immobilized on membranes showed a decrease of $12.5 \%$ after 5 cycles used in the hydrolysis of olive oil, ${ }^{7}$ and when immobilized on polyester fibers a decrease of $75 \%$ after 10 batches. $^{33}$

\section{Conclusion}

A new approach for enzymatic reactions catalyzed by lipases involving complex substrates like vegetable oils was proposed. A combi-lipase biocatalyst improved the reaction rate when compared to each lipase alone. For the hydrolysis of soybean oil, the best combi-lipase biocatalyst is composed of $80 \%$ of RML and $20 \%$ of CALB. TLL, despite being the more active lipase, did not improve the properties of the combi-lipase biocatalyst. The possibility of using a collection of a biocatalyst from the same lipase with changed properties may be a next step in this research to evaluate the real impact that it may have in the design of these reactions. This new concept may be a very useful technology for food industries in the hydrolysis of vegetable oils.

\section{Acknowledgements}

This work was supported by grants from Fundação de Amparo a Pesquisa do Rio Grande do Sul (FAPERGS; ARD/2011, Brazil), from CNPq (Brazilian Bureau of Science and Technology), and CTQ2009-07568 from Spanish Ministerio de Ciencia e Innovación. The authors would like to thank $\mathrm{Mr}$ Ramiro Martínez (Novozymes, Spain) for kindly supplying the enzymes used in this research. We also thank CNPq for a fellowship to A.M. Silva and FAPERGS for a fellowship to J. S. Alves.

\section{References}

1 D. Goswami, J. K. Basu and S. De, Crit. Rev. Biotechnol., 2013, 33, 81-96.

2 V. R. Murty, J. Bhat and P. K. A. Muniswaran, Biotechnol. Bioprocess Eng., 2002, 7, 57-66.

3 J. A. Awadallak, F. Voll, M. C. Ribas, C. Da Silva, L. C. Filho and E. A. Da Silva, Ultrason. Sonochem., 2013, 20, 1002-1007.

4 J. Čech, W. Schrott, Z. Slouka, M. Přibyl, M. Brož, G. Kuncová and D. Šnita, Biochem. Eng. J., 2012, 67, 194-202.

5 G. Fernández-Lorente, C. Pizarro, D. López-Vela, L. Betancor, A. V. Carrascosa, B. Pessela and J. M. Guisan, J. Am. Oil Chem. Soc., 2011, 88, 819-826.

6 K. M. Gonçalves, F. K. Sutili, S. G. F. Leite, R. O. M. A. De Souza and I. C. R. Leal, Ultrason. Sonochem., 2012, 19, 232-236.

7 S. Gupta, P. Ingole, K. Singh and A. Bhattacharya, J. Appl. Polym. Sci., 2012, 124, E17-E26.

8 A. Sharma, S. P. Chaurasia and A. K. Dalai, Catal. Today, 2013, 207, 93-100.

9 W. C. Ko, H. J. Wang, J. S. Hwang and C. W. Hsieh, J. Agric. Food Chem., 2006, 54, 1849-1853.

10 X. X. Pan, L. Xu, Y. Zhang, X. Xiao, X. F. Wang, Y. Liu, H. J. Zhang and Y. J. Yan, J. Agric. Food Chem., 2012, 60, 9673-9679.
11 S. A. Teichert and C. C. Akoh, J. Agric. Food Chem., 2011, 59, 9588-9595.

12 D. H. Lee, J. M. Kim, H. Y. Shin, S. W. Kang and S. W. Kim, Biotechnol. Bioprocess Eng., 2006, 11, 522-525.

13 F. Guan, P. Peng, G. Wang, T. Yin, Q. Peng, J. Huang, G. Guan and Y. Li, Process Biochem., 2010, 45, 1677-1682.

14 J. H. Lee, D. H. Lee, J. S. Lim, B. H. Um, C. Park, S. W. Kang and S. W. Kim, J. Microbiol. Biotechnol., 2008, 18, 1927-1931.

15 R. C. Rodrigues and M. A. Z. Ayub, Process Biochem., 2011, 46, 682-688.

16 E. M. Anderson, K. M. Larsson and O. Kirk, Biocatal. Biotransform., 1998, 16, 181-204.

17 R. J. Gouka, P. J. Punt, J. G. M. Hessing and C. A. M. J. J. Van Den Hondel, Appl. Environ. Microbiol., 1996, 62, 1951-1957.

18 W. Prathumpai, S. J. Flitter, M. McIntyre and J. Nielsen, Appl. Microbiol. Biotechnol., 2004, 65, 714-719.

19 M. W. Christensen, O. Kirk and C. Pedersen, US Pat. US20030203457, 2003.

20 L. Peng, X. Xu, H. Mu, C. E. Høy and J. Adler-Nissen, Enzyme Microb. Technol., 2002, 31, 523-532.

21 R. Fernandez-Lafuente, J. Mol. Catal. B: Enzym., 2010, 62, 197-212.

22 B. Huge-Jensen, D. R. Galluzzo and R. G. Jensen, Lipids, 1987, 22, 559-565.

23 B. Huge-Jensen, D. R. Galluzzo and R. G. Jensen, J. Am. Oil Chem. Soc., 1988, 65, 905-910.

24 C. Miller, H. Austin, L. Posorske and J. Gonzlez, J. Am. Oil Chem. Soc., 1988, 65, 927-931.

25 R. C. Rodrigues and R. Fernandez-Lafuente, J. Mol. Catal. B: Enzym., 2010, 64, 1-22.

26 R. C. Rodrigues and R. Fernandez-Lafuente, J. Mol. Catal. B: Enzym., 2010, 66, 15-32.

27 P. Xu, Z.-Y. Ding, Z. Qian, C.-X. Zhao and K.-C. Zhang, Enzyme Microb. Technol., 2008, 42, 325-331.

28 E. Séverac, O. Galy, F. Turon, C. A. Pantel, J. S. Condoret, P. Monsan and A. Marty, Enzyme Microb. Technol., 2011, 48, 61-70.

29 G. Fernandez-Lorente, Z. Cabrera, C. Godoy, R. FernandezLafuente, J. M. Palomo and J. M. Guisan, Process Biochem., 2008, 43, 1061-1067.

30 O. Barbosa, C. Ortiz, R. Torres and R. Fernandez-Lafuente, J. Mol. Catal. B: Enzym., 2011, 71, 124-132.

31 C. Garcia-Galan, A. Berenguer-Murcia, R. FernandezLafuente and R. C. Rodrigues, Adv. Synth. Catal., 2011, 353, 2885-2904.

32 C. Mateo, J. M. Palomo, G. Fernandez-Lorente, J. M. Guisan and R. Fernandez-Lafuente, Enzyme Microb. Technol., 2007, 40, 1451-1463.

33 M. Yigitoglu and Z. Temoçin, J. Mol. Catal. B: Enzym., 2010, 66, 130-135.

34 V. K. Rathod and A. B. Pandit, Biochem. Eng. J., 2009, 47, 9399.

35 R. C. Rodrigues, G. Volpato, K. Wada and M. A. Z. Ayub, J. Am. Oil Chem. Soc., 2008, 85, 925-930.

36 J. K. Poppe, C. Garcia-Galan, C. R. Matte, R. FernandezLafuente, R. C. Rodrigues and M. A. Z. Ayub, J. Mol. Catal. B: Enzym., 2013, 94, 51-56. 